\title{
Research Article \\ Strictly Cyclic Functionals, Reflexivity, and Hereditary Reflexivity of Operator Algebras
}

\author{
Quanyuan Chen ${ }^{1,2}$ and Xiaochun Fang ${ }^{1}$ \\ ${ }^{1}$ Department of Mathematics, Tongji University, Shanghai 200092, China \\ ${ }^{2}$ College of Information, Jingdezhen Ceramic Institute, Jingdezhen, Jiangxi 333403, China
}

Correspondence should be addressed to Quanyuan Chen, cqy0798@163.com

Received 25 September 2011; Revised 4 March 2012; Accepted 14 March 2012

Academic Editor: Gerd Teschke

Copyright (C) 2012 Q. Chen and X. Fang. This is an open access article distributed under the Creative Commons Attribution License, which permits unrestricted use, distribution, and reproduction in any medium, provided the original work is properly cited.

This paper is concerned with strictly cyclic functionals of operator algebras on Banach spaces. It is shown that if $X$ is a reflexive Banach space and $\mathcal{A}$ is a norm-closed semisimple abelian subalgebra of $B(X)$ with a strictly cyclic functional $f \in X^{*}$, then $\mathcal{A}$ is reflexive and hereditarily reflexive. Moreover, we construct a semisimple abelian operator algebra having a strictly cyclic functional but having no strictly cyclic vectors. The hereditary reflexivity of an algbra of this type can follow from theorems in this paper, but does not follow directly from the known theorems that, if a strictly cyclic operator algebra on Banach spaces is semisimple and abelian, then it is a hereditarily reflexive algebra.

\section{Introduction}

Throughout this paper, $X$ is a complex Banach space, $B_{X}$ stands for the closed unit ball of $X$ and $X^{*}$ is the dual space of $X$, the space of all continuous linear functionals on $X$. For a bounded operator $A$ on $X$, denote by Lat $A$ the lattice of all closed invariant subspaces of $A$ and $A^{*}$ the adjoint operator of $A$. For a subalgebra $A$ of $B(X)$, the Banach algebra of all bounded linear operators on $X$, denote by Lat $(\mathcal{A})$ the lattice of all closed subspaces invariant under every operator in $\mathcal{A}$. For a set $\mathcal{L}$ of subspaces of $X$, denote by $\operatorname{Alg} \mathcal{L}$ the algebra of all operators in $B(X)$ which leave all subspaces in $\mathcal{L}$ invariant. An operator algebra $\mathcal{A}$ is reflexive if

$$
\mathcal{A}=\text { AlgLat } \mathcal{A}, \quad \text { where AlgLat } \mathcal{A}=\{T \in B(X): \text { Lat } \mathscr{A} \subset \operatorname{Lat} T\} .
$$

In $[1,2]$, Loginov and Sulman introduced the following notion of a reflexive subspace of $B(X)$. If $\mathcal{S}$ is a subspace (linear manifold) of $B(X)$, we denote by Ref $\mathcal{S}$ the set 
$\{A: A \in B(X)$ and $A(x) \in \overline{\mathcal{S}(x)}$ for all $x \in X\}$, where the bar denotes norm closure. The operator subspace $\mathcal{S}$ is reflexive if $\operatorname{Ref} \mathcal{S}=\mathcal{S}$. If $\mathcal{S}$ is a subalgebra of $B(X)$ containing the identity operator $I$, then $\operatorname{Ref} \mathcal{S}=\operatorname{AlgLat} \mathcal{S}$, and $\mathcal{S}$ is reflexive as an operator subspace if and only if $\mathcal{S}$ is reflexive as an operator algebra.

In general theory of operator algebras, reflexive algebras seem to play a role somewhat analogous to the role of von Neumann algebras in the study of *-algebras. The problem of finding the conditions under which a (weakly) closed operator algebra is reflexive has an obvious connection with the problem of existence of invariant subspaces for a bounded linear operator or for an operator algebra. A concrete operator algebra of $B(X)$ is reflexive if and only if it is equivalent to $\operatorname{Alg} \mathcal{L}$ for some subspace lattice $\mathcal{L}$ of $X$. The reflexivity of an abstract operator algebra has also been studied extensively. Finding conditions for an abstract operator algebra to be reflexive has been the important concern of many scholars and there have been many papers written on the topic. See [1-14] and references therein. In this paper, we are interested in the hereditary reflexivity of operator algebras having strictly cyclic functionals.

Let $A$ be a subalgebra of $B(X)$. If there is a vector $x_{0} \in X$ such that the map $\alpha$ of $\mathcal{A}$ to $\mathcal{A} x_{0}$ by $\alpha(A)=A x_{0}$ is injective on $\mathcal{A}$, then the vector $x_{0}$ is called a separating vector for $\mathcal{A}$. Let $f_{0} \in X^{*}$. If the map $\beta_{f_{0}}$ of $\mathcal{A}$ to $X^{*}$ by $\beta_{f_{0}}(A)=A^{*}\left(f_{0}\right)$ is injective on $\mathcal{A}$, then the functional $f_{0}$ is called a separating functional for $\mathcal{A}$. Furthermore, if the subset $\left\{A^{*}\left(f_{0}\right): A \in \mathscr{A}\right\}$ is normclosed in $X^{*}$, then the functional $f_{0}$ is called a strictly separating functional for $\mathscr{A}$.

$\mathcal{A}$ is called cyclic if $\mathscr{A} x_{0}=\left\{A x_{0}: A \in \mathscr{A}\right\}$ is norm-dense in $X$ for some vector $x_{0}$ in $X$. $\mathcal{A}$ is called strictly cyclic if $A x_{0}=X$ for some vector $x_{0}$ in $X$. The vector $x_{0}$ is called cyclic vector for $\mathcal{A}$ in the former case and strictly cyclic vector in the latter. Alan Lambert proved [10] that a commutative semisimple strictly cyclic Banach algebra of operators on a Hilbert space is reflexive, and that every strictly cyclic vector for an abelian algebra is a separating vector. Later, Hadwin [7] gave a simple proof that a commutative semisimple strictly cyclic algebra of operators on a Banach space is reflexive. Meanwhile, Hadwin and Nordgren [6] proved that a reflexive operator algebra on a Banach space with a relative strictly separating vector is hereditarily reflexive (a concept initiated and studied by Loginov and Sulman [12]). In [13], Costel Peligrad explicitly stated and proved differently that if a strictly cyclic operator algebra on a Banach space is semisimple and abelian, then it is reflexive and hereditarily reflexive. In the present paper, a similar result is obtained without the hypothesis that the operator algebra $\mathcal{A}$ is strictly cyclic, provided that $\mathbb{A}$ has a strictly cyclic functional. Moreover, we construct a semisimple single-generated algebra $\mathbb{A}$ having a strictly cyclic functional but having no strictly cyclic vectors. An algebra of this type is reflexive by Theorem 3.8 and hereditarily reflexive by Theorem 3.12. However, the (hereditary) reflexivity of an algebra of this type does not follow directly from the results above.

The paper is organized as follows. In Section 2, we introduce the notion of a (strictly) cyclic functional for operator algebras on a Banach space and develop some properties of strictly cyclic functionals. In Section 3, we clarify the role of the strictly cyclic functional in the reflexivity of operator algebra. The main results of Section 3 are Theorems 3.8 and 3.12. It is shown that a semisimple abelian subalgebra of $B(X)$ with a strictly cyclic functional $f_{0}$ is reflexive and hereditarily reflexive when $X$ is a reflexive Banach space. The reflexivity of some operator algebras on Banach spaces can follow from Theorem 3.8. In Section 4, we construct a semisimple single-generated algebra $\mathcal{A}$ having a strictly cyclic functional but having no strictly cyclic vectors. An algebra of this type is reflexive by Theorem 3.8 and hereditarily reflexive by Theorem 3.12. However, the (hereditary) reflexivity of an algebra of this type does not follow directly from the theorems of Costel Peligrad. 


\section{Strict Cyclic Functionals}

Definition 2.1. Let $X$ be a Banach space and let $S$ be a subalgebra or a subspace of $B(X)$. Let $X^{*}$ be the dual space of $X$. If there is a functional $f_{0} \in X^{*}$ such that

$$
\mathcal{S}^{*} f_{0}=\left\{A^{*}\left(f_{0}\right): A \in \mathcal{S}\right\}=\left\{\left(f_{0} \circ A\right): A \in \mathcal{S}\right\}
$$

is norm-dense in $X^{*}$, then $f_{0}$ is called a cyclic functional for $\mathcal{S}$. If there is a functional $f_{0} \in X^{*}$ such that

$$
\mathcal{S}^{*} f_{0}=\left\{A^{*}\left(f_{0}\right): A \in \mathcal{S}\right\}=\left\{\left(f_{0} \circ A\right): A \in \mathcal{S}\right\}=X^{*},
$$

then $f_{0}$ is called a strictly cyclic functional for $\mathcal{S}$.

Lemma 2.2 (Open Mapping Lemma). Suppose that $X$ is a Banach space and $Y$ is a normed linear space, and $\varepsilon_{f_{0}}: X \rightarrow Y$ is a bounded operator. Assume that there exist $k>0$ and $0<\eta<1$ such that

$$
B_{Y} \subseteq k \cdot \varepsilon_{f_{0}}\left(B_{X}\right)+\eta B_{Y}
$$

Then, $\varepsilon_{f_{0}}$ is a surjective open map, and $Y$ is complete.

Proposition 2.3. Let $A$ be a norm-closed subalgebra of $B(X)$ and $f_{0} \in X^{*}$ with $f_{0} \neq 0$. Then, the following are equivalent:

(1) $f_{0}$ is a strictly cyclic functional for $\mathrm{A}$;

(2) there exists a constant $C>0$ such that for all $g \in B_{X^{*}}$ there is an operator $A \in \mathcal{A}$ with $\|A\| \leqslant C$ such that $A^{*}\left(f_{0}\right)=g$;

(3) there are $C>0$ and $0<\eta<1$ such that for all $g \in B_{X^{*}}$ there is an operator $A \in \mathcal{A}$ with $\|A\| \leqslant C$ such that $\left\|g-A^{*}\left(f_{0}\right)\right\|<\eta$.

Proof. Define $\varepsilon_{f_{0}}: \mathcal{A} \rightarrow X^{*}$ by $\varepsilon_{f_{0}}(A)=A^{*}\left(f_{0}\right)$ for $A \in \mathcal{A}$, then $\varepsilon_{f_{0}}$ is a bounded linear operator from $A$ to $X^{*}$. Since $\mathcal{A}$ is norm-closed, it can be viewed as a Banach space. Thus, the implication $(1) \Rightarrow(2)$ follows immediately from Open Mapping Theorem. The implication (2) $\Rightarrow(3)$ is trivial. Finally, the implication $(3) \Rightarrow(1)$ follows from Open Mapping Lemma.

The following proposition is similar to Theorem 2 in [15].

Proposition 2.4. Let $A$ be a norm-closed subalgebra of $B(X)$ with a strictly cyclic functional $f_{0} \in X^{*}$.

(1) Then, every cyclic functional $f$ for $\mathcal{A}$ is actually a strictly cyclic functional for $\mathcal{A}$.

(2) Moreover, the set of all (strictly) cyclic functionals for $\mathbb{A}$ is norm-open in $X^{*}$ and coincides with

$$
\mathcal{C}_{\mathcal{A}}=\left\{A^{*}\left(f_{0}\right): A \in \mathcal{A} \text { and } A^{*} \text { has a left inverse }\left(\bmod \operatorname{ker}\left(\varepsilon_{f_{0}}\right)\right) \text { in } \mathcal{A}^{*}\right\},
$$

where $\varepsilon_{f_{0}}$ is the map from $\mathcal{A}$ onto $X^{*}$ by $\varepsilon_{f_{0}}(A)=A^{*}\left(f_{0}\right)$ for every $A \in \mathcal{A}$, and $\operatorname{ker}\left(\varepsilon_{f_{0}}\right)$ is the kernel of the map $\varepsilon_{f_{0}}$. 
Proof. (1) If $f$ is a cyclic functional for $\mathcal{A}$, then $N=\mathcal{A}^{*} f=\left\{A^{*}(f): A \in \mathcal{A}\right\}$ is a normdense linear manifold of $X^{*}$ and $N$ is $\mathcal{A}^{*}$-invariant. We will show that $f_{0} \in N$ and thus $X^{*}=\mathscr{A}^{*}\left(f_{0}\right)=\left\{A^{*}\left(f_{0}\right): A \in \mathscr{A}\right\} \subseteq \mathscr{A}^{*} N \subseteq N$.

Let $f_{n}$ be a sequence in $N$ such that $\left\{f_{n}\right\}$ converges uniformly to $f_{0}$. By Proposition 2.3 (3), we may assume the existence of sequence $A_{n}$ such that $A_{n}{ }^{*}\left(f_{0}\right)=f_{0}-f_{n}$ and $\lim _{n \rightarrow \infty}\left\|A_{n}\right\|=0$. Thus, for $n$ sufficiently large, $\left\|A_{n}{ }^{*}\right\|<1$ and $\left(I-A_{n}{ }^{*}\right)^{-1}=\sum_{k=0}^{\infty}\left(A_{n}{ }^{*}\right)^{k}=$ $\sum_{k=0}^{\infty}\left(A_{n}{ }^{k}\right)^{*}$. Therefore, $\left(I-A_{n}{ }^{*}\right)^{-1} \in \mathcal{A}^{*}$ and since $\left(I-A_{n}{ }^{*}\right) f_{0}=f_{n}$, we have $f_{0}=\left(I-A_{n}{ }^{*}\right)^{-1}$ $f_{n} \in N$.

(2) Choose $C$ as in Proposition 2.3 (2). Let $0<\delta<1 / C$ and suppose that $f \in X^{*}$ with $\left\|f-f_{0}\right\|<\delta$. Now if $g \in B_{X^{*}}$, we choose $A \in \mathcal{A}$ with $\|A\| \leqslant C$ and $A^{*}\left(f_{0}\right)=g$. But then, $\left\|A^{*}(f)-g\right\|=\left\|A^{*}(f)-A^{*}\left(f_{0}\right)\right\| \leqslant C \delta<1$. Hence, $f$ is a strictly cyclic functional for the algebra $A$ by Proposition 2.3 (3). Thus, the set of all (strictly) cyclic functionals for $A$ is uniformly open in $X^{*}$.

Clearly, if $g=A^{*}\left(f_{0}\right) \in \mathcal{C}_{A}$, then there exists $B \in \mathcal{A}$ such that $B^{*} A^{*}=I+L$ for some $L \in \operatorname{ker}\left(\varepsilon_{f_{0}}\right)$. Therefore, $f_{0}=B^{*} A^{*}\left(f_{0}\right) \in \mathcal{A}^{*}\left(f_{0}\right)$. Hence, $X^{*}=\mathscr{A}^{*} f_{0} \subseteq \mathcal{A}^{*} g$, which implies that $g$ is a strictly cyclic functional for $\mathcal{A}$.

Conversely, if $g=A^{*}\left(f_{0}\right)$ is a cyclic (and, therefore, it is strictly cyclic) functional for $\mathcal{A}$, then $f_{0} \in X^{*}=\left\{B^{*} A^{*}\left(f_{0}\right): B \in \mathcal{A}\right\}$. Therefore, there exists $B \in \mathcal{A}$ such that $B^{*} A^{*}\left(f_{0}\right)=f_{0}$, that is, $\left(B^{*} A^{*}-I\right) f_{0}=0$. Hence, $B^{*} A^{*}-I=L \in \operatorname{ker}\left(\varepsilon_{f_{0}}\right)$ and $B^{*} A^{*}=I-L$. Therefore, $B^{*}$ is a left inverse of $A^{*}$ module $\operatorname{ker}\left(\varepsilon_{f_{0}}\right)$. Hence, $g=A^{*}\left(f_{0}\right) \in \mathcal{C}_{A}$.

Remark 2.5. If $\mathcal{A}$ is a norm-closed commutative subalgebra of $B(X)$ with a strictly cyclic functional $f_{0} \in X^{*}$, then $I \in \mathcal{A}$. Indeed, let $A_{0} \in \mathcal{A}$ such that $A_{0}^{*}\left(f_{0}\right)=f_{0}$. Then, $A^{*} A_{0}^{*}\left(f_{0}\right)=$ $A^{*}\left(f_{0}\right)$ for every $A \in \mathcal{A}$. Hence, by commutativity of $\mathcal{A}, A_{0}^{*} A^{*}\left(f_{0}\right)=A^{*}\left(f_{0}\right)$ for every $A \in \mathcal{A}$. Therefore, $A_{0}^{*}$ is the identity operator on $X^{*}$ and $A_{0}=I \in \mathscr{A}$.

\section{Hereditary Reflexivity and Strictly Cyclic Functional}

Remark 3.1. Let $X$ be a Banach space and let $S$ be a subalgebra or a subspace of $B(X)$. Then, (1) $f_{0} \in X^{*}$ is a strictly cyclic functional for $\mathcal{S}$ if and only if $f_{0}$ is a strictly cyclic vector for $\mathcal{S}^{*}=\left\{T^{*}: T \in \mathcal{S}\right\}$. $T \in \mathcal{S}\}$.

(2) $f_{0} \in X^{*}$ is a cyclic functional for $\mathcal{S}$ if and only if $f_{0}$ is a cyclic vector for $\mathcal{S}^{*}=\left\{T^{*}\right.$ :

Lemma 3.2. (1) Suppose that $X$ is a Banach space which is not a Hilbert space. If $T \in B(X)$, then $\sigma\left(T^{*}\right)=\sigma(T)$.

(2) Suppose that $X$ is a Hilbert space. If $T \in B(X)$, then $\sigma\left(T^{*}\right)=\{\bar{\lambda}: \lambda \in \sigma(T)\}$.

Lemma 3.3. Suppose that $X$ is a Banach space and $\mathcal{A}$ is an abelian subalgebra of $B(X)$. Set $\mathscr{A}^{*}=$ $\left\{A^{*}: A \in \mathcal{A}\right\}$. Then, the Banach algebra $\mathcal{A}^{*}$ is semisimple if and only if the Banach algebra $\mathcal{A}$ is semisimple.

Proof. We may assume that $\mathcal{A}$ is unital for convenience. Let $\mathcal{A}^{*}$ be semisimple and $T \in$ $\mathcal{A}(T \neq 0)$. Then, $T^{*} \neq 0$ and there is a multiplicative linear functional $F$ on $\mathscr{A}^{*}$ such that $F\left(T^{*}\right) \neq 0$. Since $\sigma\left(T^{*}\right)=\left\{F\left(T^{*}\right): F\right.$ is a multiplicative linear functional on $\left.\mathscr{A}^{*}\right\}$ by Gelfand transformation, $\sigma\left(T^{*}\right)$ contains a nonzero scalar and $\sigma(T)$ contains a nonzero scalar by Lemma 3.2. Since $\sigma(T)=\{G(T): G$ is a multiplicative linear functional on $\mathbb{A}\}$, it follows 
that there is a multiplicative linear functional $G$ on $\mathcal{A}$ such that $G(T) \neq 0$. Hence, $\mathscr{A}$ is also semisimple. Similarly, if $\mathscr{A}$ is semisimple, then $\mathscr{A}^{*}$ is also semisimple.

Let $X$ be a Banach space, and let $M$ be a nonempty subset of $X$ and $N$ be a nonempty subset of $X^{*}$. The annihilator $M^{\perp}$ of $M$ and the preannihilator ${ }^{\perp} N$ of $N$ are defined as follows [16]: $M^{\perp}=\left\{f \in X^{*}: f(x)=0\right.$ for all $\left.x \in M\right\},{ }^{\perp} N=\{x \in X: f(x)=0$ for all $f \in N\}$. It is obvious that $M^{\perp}$ is a weak ${ }^{*}$-closed subspace of $X^{*}$ and ${ }^{\perp} N$ is a norm-closed subspace of $X .{ }^{\perp}\left(M^{\perp}\right)$ is the norm-closure of $M$ in $X$ and $\left({ }^{\perp} N\right)^{\perp}$ is the weak ${ }^{*}$-closure of $N$ in $X^{*}$.

Lemma 3.4. Suppose that $X$ is a Banach space and $M$ is a closed subspace of $X$ and $N$ is a normclosed subspace of $X^{*}$. Then,

(1) ${ }^{\perp}\left(M^{\perp}\right)=M$;

(2) furthermore, if $\mathrm{X}$ is a reflexive Banach space, then $\left({ }^{\perp} N\right)^{\perp}=N$.

Proof. (1) It is clear.

(2) Let $X$ be a reflexive Banach space and let $J$ be the natural imbedding map from $X$ into $X^{* *}$. First, we observe that $\left({ }^{\perp} N\right)^{\perp}$ is the weak ${ }^{*}$-closure of $N$ in $X^{*}$ that includes $N$, that is, $N \subseteq\left({ }^{\perp} N\right)^{\perp}$.

Now, we suppose that there exists $x_{0}^{*} \in\left({ }^{\perp} N\right)^{\perp}$ but $x_{0}^{*} \notin N$. Then, there exists $x^{* *} \in X^{* *}$ such that $x^{* *}\left(x_{0}^{*}\right)=1$ and $x^{* *}(f)=0$ for any $f \in N$. Since there exists $x \in X$ such that $J x=x^{* *}$ by the reflexivity of the Banach space $X$, we have that $x_{0}^{*}(x)=1$ and $f(x)=0$ for any $f \in N$. It follows that $x_{0}^{*}(x)=1$ and $x_{0}^{*} \notin\left({ }^{\perp} N\right)^{\perp}$, which is a contradiction to $x_{0}^{*} \in\left({ }^{\perp} N\right)^{\perp}$. The proof is complete.

Lemma 3.5. Let $A$ be a bounded linear operator on a reflexive Banach space $X$. Then, $N \in$ Lat $A^{*}$ if and only if ${ }^{\perp} N \in$ Lat $A$.

Proof. If ${ }^{\perp} N \in$ Lat $A$, then, for any $f$ in $N$ and $x$ in ${ }^{\perp} N$, we have that $A x \in\left({ }^{\perp} N\right)$ and $\left(A^{*} f\right)(x)=f(A x)=0$. Thus, $A^{*}(f) \in\left({ }^{\perp} N\right)^{\perp}=N$ by Lemma 3.4. Hence, $N \in \operatorname{Lat}\left(A^{*}\right)$.

Conversely, if $N \in \operatorname{Lat}\left(A^{*}\right)$, then for any $x$ in ${ }^{\perp} N$ and $f$ in $N$, we have $A^{*} f \in N$ and $f(A x)=\left(A^{*} f\right)(x)=0$. Thus $A x \in\left({ }^{\perp} N\right)$. So ${ }^{\perp} N \in \operatorname{Lat} A$.

Lemma 3.6. Let $X$ be a reflexive Banach space and let $\mathcal{A}$ be a subalgebra of $B(X)$. Let $\mathscr{A}^{*}=\left\{A^{*}\right.$ : $A \in \mathcal{A}\}$. If $\mathcal{A}^{*}$ is reflexive, then $\mathcal{A}$ is a reflexive subalgebra.

Proof. Let $\mathcal{A}^{*}$ be reflexive. Suppose that $T$ is a bounded operator leaving invariant all the closed invariant subspaces of $\mathcal{A}$, that is, Lat $\mathcal{A} \subset$ Lat $T$. For each norm-closed invariant subspace $N\left(N \subseteq X^{*}\right)$ of $\mathscr{A}^{*}$, that is, $N \in$ Lat $^{*}{ }^{*}$, we have that ${ }^{\perp} N \in$ Lat $\mathscr{A}$. Since Lat $\mathscr{A} \subset$ Lat $T,{ }^{\perp} N \in \operatorname{Lat} T$. It follows that $N \in \operatorname{Lat}\left(T^{*}\right)$ by Lemma 3.5. Thus, Lat $\mathscr{A}^{*} \subset$ Lat $T^{*}$. Since $\mathscr{A}^{*}$ is reflexive, $T^{*} \in \mathcal{A}^{*}$. It follows that $T \in \mathcal{A}$ and $\mathcal{A}$ is reflexive.

Lemma 3.7 ([7, Theorem 8] and [1, 2, Theorem 6]). Suppose that X is a Banach space and $\mathcal{A}$ is an abelian subalgebra of $B(X)$. If $\mathbb{A}$ is a norm-closed, semisimple, strictly cyclic algebra, then $\mathbb{A}$ is reflexive.

Theorem 3.8. Let $X$ be a reflexive Banach space and let $\mathcal{A}$ be an abelian subalgebra of $B(X)$ with a strictly cyclic functional $f \in X^{*}$. If $A$ is a norm-closed semisimple algebra, then $A$ is reflexive. 
Proof. If $f$ is a strictly cyclic functional for $\mathcal{A}$, then $f$ is a strictly cyclic vector for $\mathscr{A}^{*}$ by Remark 3.1. Since $\mathcal{A}$ is semisimple, $\mathscr{A}^{*}$ is also semisimple by Lemma 3.3. It follows that $\mathscr{A}^{*}$ is reflexive by Lemma 3.7. Thus, $\mathcal{A}$ is reflexive by Lemma 3.6.

Definition $3.9([1,2,12])$. A norm-closed subalgebra $A$ of $B(X)$ is called hereditarily reflexive if, for every weakly closed subspace $\mathcal{L} \subseteq \mathcal{A}$, every $x \in X$ and $T \in B(X), T x \in \overline{\mathcal{L} x}$ implies $T \in \mathcal{L}$. Here, $\overline{\mathcal{L} x}$ denotes the norm closure of $\{L x \mid L \in \mathcal{L}\}$ in $X$.

Lemma 3.10. Let $X$ be a reflexive Banach space and let $A$ be a unital abelian subalgebra of $B(X)$. Let $\mathscr{A}^{*}=\left\{A^{*}: A \in \mathscr{A}\right\}$. Then, $\mathbb{A}$ is hereditarily reflexive if and only if $\mathscr{A}^{*}$ is hereditarily reflexive.

Proof. If $X$ is a reflexive Banach space, then we only need to show that the hereditary reflexivity of $\mathscr{A}$ implies the hereditary reflexivity of $\mathscr{A}^{*}$.

Let $\mathscr{A}$ be hereditarily reflexive. Let $\mathcal{L}^{*} \subseteq \mathcal{A}^{*}$ be a norm closed subspace of $\mathscr{A}^{*}$ and $T^{*} \in$ $B\left(X^{*}\right)$. We suppose that $T^{*}(f) \in \overline{\mathcal{L}^{*}(f)}$ for every continuous functional $f \in X^{*}$. Then, for any given $x \in X, T^{*} f(x) \in \overline{\mathcal{L}^{*}(f)}(x)$. Here, $\overline{\mathcal{L}^{*}(f)}$ denotes the norm closure of $\left\{L^{*}(f) \mid L \in \mathcal{L}\right\}$ in $X^{*}$. Let $g \in(\overline{\mathcal{L} x})^{\perp}$. Then, $\left(T^{*} g\right)(x) \in \overline{\mathcal{L}^{*} g}(x)$ for any $x \in X$. Since $\left(L^{*} g\right)(x)=g(L x)=0$ for any $L \in \mathcal{L}$, we have that $g(T x)=\left(T^{*} g\right)(x)=0$. It follows that $T x \in^{\perp}\left((\overline{\mathcal{L} x})^{\perp}\right)=\overline{\mathcal{L} x}$ by Lemma 3.4. Thus, $T \in \mathcal{L}$ by the hereditary reflexivity of $\mathcal{A}$. It follows that $T^{*} \in \mathcal{L}^{*}$ and $\mathcal{A}^{*}$ are hereditarily reflexive.

Lemma 3.11 ([10, Theorem 10], and [1, 2, Corollary 2.5]). Suppose that $X$ is a Banach space and $A$ is an abelian subalgebra of $B(X)$. If $A$ is a norm-closed, semisimple, strictly cyclic subalgebra, then A is hereditarily reflexive.

Theorem 3.12. Let $X$ be a reflexive Banach space and let $A$ be an abelian subalgebra of $B(X)$ with a strictly cyclic functional $f \in X^{*}$. If $\mathbb{A}$ is a norm-closed semisimple algebra, then $\mathbb{A}$ is hereditarily reflexive.

Proof. The proof is similar to the proof of Theorem 3.8.

The following proposition is similar to Theorem 2.3 in [1, 2].

Proposition 3.13. Let $X$ be a Banach space. A subspace $S \subseteq B(X)$ is hereditarily reflexive if and only if it is reflexive and every weakly continuous functional on $\mathcal{S}$ is of the form $A \mapsto f(A(x))(A \in \mathcal{S})$, where $x \in X$ and $f \in X^{*}$.

Proof. If $\mathcal{S}$ is hereditarily reflexive, then $\mathcal{S}$ is reflexive by definition. Let $F$ be a weakly continuous functional on $\mathcal{S}$ and let $\mathcal{K}_{F}=\operatorname{ker} F$, then $\mathcal{K}_{F}$ is a reflexive subspace. Choose $B \in \mathcal{S}$ such that $F(B)=1$. Since $B \notin \mathcal{K}_{F}=\operatorname{Ref} \mathcal{K}_{F}$, there exists a vector $x \in X$ such that $\left\|\left(B-A_{0}\right)(x)\right\|>1=F(B)$ for all $A_{0} \in \mathcal{K}_{F}$. Since $F(B)=F\left(B-A_{0}\right)$ for any $A_{0} \in \mathcal{K}_{F}$, by linearity we have the inequality $\|A x\| \geqslant F(A)$ for all $A \in \mathcal{S}$. Define the linear functional $\phi$ on $\mathcal{S} x$ by $\phi(A x)=F(A)(A \in \mathcal{S})$. Then, $|\phi(A x)| \leqslant\|A x\|$, that is, $\phi$ is well defined and is continuous; thus there exists $f \in X^{*}$ such that $F(A)=\phi(A x)=f(A(x))$ for all $A \in \mathcal{S}$.

We now prove sufficiency. Let $F$ be a weakly continuous functional on $\mathcal{S}$ such that $F(A)=f(A(x))$ for all $A \in \mathcal{S}$. Clearly, $\mathcal{K}_{F}=\operatorname{ker} F$ is a reflexive operator subspace. By HahnBanach Theorem, every weakly closed subspace of $\mathcal{S}$ is the intersection of the kernels of weakly continuous functionals. Since the intersection of any family of reflexive subspaces is reflexive, we have that $\mathcal{S}$ is hereditarily reflexive. 
Lemma 3.14. Let $X$ be a Banach space and let $\mathcal{A}$ be an abelian subalgebra of $B(X)$ with a strictly cyclic functional $f_{0} \in X^{*}$. Then, the dual space of $A$ consists entirely of the maps $A \mapsto F\left(A^{*} f_{0}\right)(A \in$ A), where $F \in X^{* *}$.

Proof. For any $F \in X^{* *}$, the map $A \mapsto F\left(A^{*} f_{0}\right)(A \in \mathcal{A})$ defines a continuous linear functional on $A$.

Conversely, suppose that $g$ is a continuous linear functional on $\mathcal{A}$. Define the map $C: \mathcal{A} \rightarrow \mathcal{A}^{*}$ by $C(A)=A^{*}(A \in \mathcal{A})$ and define map $\rho: \mathscr{A}^{*} \rightarrow X^{*}$ by $\rho\left(A^{*}\right)=A^{*} f_{0}(A \in \mathcal{A})$ (see the graph below). Notice that both map $C$ and map $\rho$ are inverse:

$$
\mathcal{A} \stackrel{C}{\longrightarrow} \mathcal{A}^{*} \stackrel{\rho}{\longrightarrow} X^{*}, \quad A \stackrel{C}{\longrightarrow} A^{*} \stackrel{\rho}{\longrightarrow} A^{*} f_{0} .
$$

Then $\left(g \circ C^{-1} \circ \rho^{-1}\right)$ is a continuous linear functional on $X^{* *}$. It follows that there exists $F \in X^{* *}$ such that $g(A)=\left(g \circ C^{-1} \circ \rho^{-1}\right)(f)=F(f)$, where $A \in \mathcal{A}$ and $f=\rho\left(A^{*}\right)=A^{*} f_{0}$. Thus, $g(A)=$ $F\left(A^{*} f_{0}\right)$.

Theorem 3.15. Let $X$ be a Banach space and let $A$ be a semisimple abelian subalgebra of $B(X)$ with a strictly cyclic functional $f_{0} \in X^{*}$. Then, $A$ is hereditarily reflexive if and only if $X$ is a reflexive Banach space.

Proof. If $X$ is a reflexive Banach space, then $\mathcal{A}$ is hereditarily reflexive by Theorem 3.12 . Conversely, if $\mathcal{A}$ is hereditarily reflexive, then by Proposition 3.13, it is reflexive and every weakly continuous functional on $\mathcal{A}$ is of the form $A \mapsto f(A(x))(A \in \mathcal{A})$, where $x \in X$ and $f \in X^{*}$. Notice that $\mathscr{A}^{*}$ is a strictly cyclic commutative subalgebra. For any $f \in X^{*}$, there exists $A_{f} \in \mathcal{A}$ such that $A_{f}^{*} f_{0}=f$. Then,

$$
\begin{aligned}
f(A(x)) & =A^{*} f(x)=A^{*}\left(A_{f}^{*} f_{0}\right)(x) \\
& =\left(A_{f} A\right)^{*}\left(f_{0}\right)(x)=f_{0}\left(A\left(A_{f} x\right)\right) \\
& =f_{0}\left(A x_{1}\right),
\end{aligned}
$$

where $x_{1}=A_{f} x \in X$. It follows that every weakly continuous functional on $\mathscr{A}$ is of the form $A \mapsto f_{0}\left(A\left(x_{1}\right)\right)(A \in \mathscr{A})$, where $x_{1} \in X$. $\mathcal{A}^{*}$ and $\mathscr{A}$ are norm-closed. Then, $\mathscr{A}^{*}$ and $X^{*}$ are isomorphic as Banach spaces. Thus, $\mathscr{A}$ and $X^{*}$ are isomorphic as Banach spaces. It follows that the set of all weakly continuous linear functionals on $\mathcal{A}$ is the set of all norm-continuous linear functionals on $\mathcal{A}$, and by Lemma 3.14, they are of the form $A \mapsto F\left(A^{*} f_{0}\right)(A \in \mathcal{A})$, where $F \in X^{* *}$.

By Proposition 3.13, $\mathscr{A}_{S}$ is hereditarily reflexive if and only if it is reflexive and every weakly continuous functional on $\mathcal{A}$ is of the form $A \mapsto f(A(x))(A \in \mathcal{A})$, where $x \in X$ and $f \in X^{*}$. It follows that $X$ is reflexive.

\section{An Operator Algebra Having a Strictly Cyclic Functional with No Strictly Cyclic Vectors}

Throughout this section, we assume that $1<p<\infty$ and $1 / p+1 / q=1$. Let $l^{p}$ be the Banach space of all absolutely $p$-summable sequences $x=\left\{\xi_{n}\right\}_{n=0}^{\infty}$ of complex numbers with 
$\|x\|=\left(\sum_{n=0}^{\infty}\left|\xi_{n}\right|^{p}\right)^{1 / p}$. Let $c_{0}$ be the space of sequences $x=\left(\xi_{n}\right)_{n=0}^{\infty}$ such that $\lim _{n \rightarrow \infty} \xi_{n}=0$ with the norm $\|x\|=\sup _{n}\left\{\left|\xi_{n}\right|\right\} .\left\{e_{n}\right\}_{n=0}^{\infty}$ is the natural basis for $l^{p}$ or $c_{0}$, that is, $e_{n}=(0$, $0, \ldots, 0,1,0, \ldots)$ with 1 as the $n$th component (beginning the indexing at 0 ). For a sequence $\left\{w_{n}\right\}_{n=1}^{\infty}$ of nonzero complex numbers, let $T$ be the linear transformation on $l^{p}$ defined by

$$
T e_{0}=0, \quad T e_{n}=w_{n} e_{n-1}, \quad \text { for } n \geq 1
$$

Obviously $T$ is a unilateral weighted backward shift with weight sequence $\left\{w_{n}\right\}_{n=1}^{\infty}$.

Let $\left\{f_{m}\right\}_{m=0}^{\infty}$ be the natural basis for $l^{9}$, that is, $f_{m}=(0,0, \ldots, 0,1,0, \ldots)$ with 1 as the $m$ th component (beginning the indexing at 0 ). A short computation shows that the adjoint operator $T^{*}$ of $T$ is defined by $T^{*}\left(f_{m}\right)=w_{m+1} f_{m+1}$ for all $m=0,1,2, \ldots$ Obviously $T^{*}$ is a unilateral weighted forward shift with weight sequence $\left\{w_{n}\right\}_{n=1}^{\infty}$.

For an operator $T$ on a Banach space $X$, denote by $\mathcal{A}_{T}$ the weakly closed subalgebra generated by $T$ and the identity operator $I$. An operator $T$ on a Banach space is called strictly cyclic if the weakly closed subalgebra $A_{T}$ is strictly cyclic.

Lemma 4.1. Let $X$ be a Banach space and let $A$ be a subspace of $B(X)$. If $\mathcal{A}$ has a strictly cyclic separating vector $x$, then there exist $k_{1}>0$ and $k_{2}>0$ such that

$$
k_{1}\|A\| \leqslant\|A x\| \leqslant k_{2}\|A\|
$$

for any $A \in \mathcal{A}$.

Proof. Let $x$ be a strictly cyclic separating vector for $\mathcal{A}$. The evaluation map $\varepsilon_{x}: A \mapsto A x$ is bounded below and has dense range (as a mapping from the algebra $\mathcal{A}$ onto $X$ ). It follows that $\varepsilon_{x}$ and $\varepsilon_{x}^{-1}$ are both continuous. The result follows.

Remark 4.2. If $T$ is strictly cyclic, then the norm topology and the strong topology on $\mathscr{A}_{T}$ coincide. If $T$ has a strictly cyclic functional, then the norm topology and the strong topology on $\mathscr{A}_{T}^{*}$ coincide. Since $I \in \mathcal{A}_{T}^{*}, \mathcal{A}_{T}^{*}$ is also the strongly closed subalgebra generated by $T^{*}$ and $I$.

It is easy to check the following statement.

Proposition 4.3. Any unilateral weighted backward shift $T$ on $l^{p}(1<p<\infty)$ or $c_{0}$ with nonzero weights is not strictly cyclic.

Example 4.4. Let $T$ be a unilateral weighted backward shift on $l^{p}(1<p<\infty)$ with weight sequence $\left\{w_{n}\right\}_{n=1}^{\infty}: w_{n}=(n+1) / n$ for all $n \geqslant 1$. Then,

(1) $T$ is not strictly cyclic;

(2) $T^{*}$ is strictly cyclic on $l q$;

(3) The weakly closed algebra $\mathcal{A}_{T^{*}}$ generated by $T^{*}$ is semisimple. Therefore, $\mathscr{A}_{T}$ is also semisimple by Lemma 3.3;

(4) $\mathscr{A}_{T^{*}}$ is hereditarily reflexive, and it follows that $\mathscr{A}_{T}$ is hereditarily reflexive from Theorem 3.12. 
Proof. (2) Set $\beta_{0}=1, \beta_{n}=\prod_{k=1}^{n} w_{k}=(2 / 1) \cdot(3 / 2) \cdots(n+1) / n=n+1$

$$
\begin{aligned}
\sum_{m=0}^{n}\left|\frac{\beta_{n}}{\beta_{m} \beta_{n-m}}\right|^{p} & =1+\sum_{m=0}^{n}\left|\frac{n+1}{(m+1) \cdot(n-m+1)}\right|^{p} \\
& =1+\frac{(n+1)^{p}}{(n+2)^{p}} \sum_{m=1}^{n}\left|\frac{1}{m+1}+\frac{1}{n-m+1}\right|^{p} \\
& \leq 1+\sum_{m=1}^{n}\left|\frac{1}{m+1}+\frac{1}{n-m+1}\right|^{p} \\
& \leq 1+\sum_{m=1}^{n} 2^{p}\left(\frac{1}{(m+1)^{p}}+\frac{1}{(n-m+1)^{p}}\right) \\
& \leq 1+2^{p-1} \sum_{m=1}^{\infty} \frac{1}{m^{p}}=C .
\end{aligned}
$$

It follows that $\sup _{n} \sum_{m=0}^{n}\left|\beta_{n} / \beta_{m} \beta_{n-m}\right|^{p}<\infty$. Since $\left\{\left|w_{n}\right|\right\}$ is monotonically decreasing, $T^{*}$ is strictly cyclic on $l^{q}$ by [17, Theorem 3.2].

(3) It is easy to see that $\left\|T^{n}\right\|=\left\|\left(T^{*}\right)^{n}\right\|=\sup _{k \in \mathbb{N}}\left|\prod_{i=0}^{n-1} w_{k+i}\right|=\sup _{k \in \mathbb{N}}(1+n / k)=1+n$. Then, $\|T\|=\left\|T^{*}\right\|=2$ and the spectral radius $r(T)=r\left(T^{*}\right)=\lim _{n \rightarrow \infty}\left\|T^{n}\right\|^{1 / n}=\lim _{n \rightarrow \infty}(1+$ $n)^{1 / n}=1$. Since $T^{*}$ is unilateral forward shift on $l^{q}$, the spectra $\sigma(T)\left(=\sigma\left(T^{*}\right)\right)$ of $T$ contains infinite complex numbers. Notice that the elements of $\mathscr{A}_{T}^{*}$ consist of all $P\left(T^{*}\right) \mathrm{s}$ and their norm limits [18], where $P(\cdot)$ are all polynomials. Then, the elements of $\mathcal{A}_{T}$ consist of all $P(T) \mathrm{s}$ and their norm limits, where $P(\cdot)$ are all polynomials. Then, we have that the Jacobson radical $\operatorname{rad}\left(\mathscr{A}_{T}\right)=\{0\}$.

In fact, since $\mathcal{A}_{T}$ is unital,

$$
\operatorname{rad}\left(\mathscr{A}_{T}\right)=\left\{A \in \mathscr{A}_{T}: A B \text { is quasinilpotent for any } B \in \mathscr{A}_{T}\right\} .
$$

For any $A \in \mathcal{A}_{T}$, there exists a sequence of polynomials $Q_{n}(\lambda)$ such that $\left\|Q_{n}(T)-A\right\| \rightarrow 0$. Let $A_{1}=P_{1}(T), A_{2}=P_{2}(T) \in \mathcal{A}_{T}$, where $P_{1}(\cdot), P_{2}(\cdot)$ are polynomials. It is obvious that $\sigma\left(A_{1} A_{2}\right)=\sigma\left(A_{1}\right) \sigma\left(A_{2}\right)=P_{1}(\sigma(T)) \cdot P_{2}(\sigma(T))$ by Spectral Theorem. If $A_{1} A_{2}$ is quasinilpotent, then $\{0\}=\sigma\left(A_{1} A_{2}\right)=\sigma\left(A_{1}\right) \sigma\left(A_{2}\right)$. It follows that, if $A \in \operatorname{rad}\left(\mathcal{A}_{T}\right)$, then $\sigma(A) \cdot P_{2}(\sigma(T))=\{0\}$ for any polynomial $P_{2}(\lambda)$, and $\sigma(A)=\{0\}$ since $\sigma(T)$ contains infinite complex numbers. Thus, $Q_{n}(\sigma(T)) \rightarrow 0$ and $Q_{n}(T) \rightarrow 0$, that is, $A=0$. So $A_{T}$ is semisimple.

Remark 4.5. Let $T$ be a unilateral weighted backward shift on the reflexive Banach space $l^{p}$ as in Example 4.4. Then, the weakly closed algebra $\mathcal{A}_{T}$, generated by $T$ and $I$, is normclosed, semisimple, and abelian. And $\mathcal{A}_{T}$ has a strictly cyclic functional but has no strictly cyclic vectors. An algebra of this type is reflexive (hereditarily reflexive) by Theorem 3.8 (Theorem 3.12). However, the reflexivity of an algebra of this type does not follow directly from the theorem of Costel Peligrad.

Remark 4.6. If the Banach space $X$ is not reflexive, then the algebra $\mathcal{A}$ described as in Theorem 3.12 is not hereditarily reflexive. We do not know whether the reflexivity of an 
operator algebra $\mathcal{A} \subseteq B(X)$ described as in Theorem 3.8 is independent of the reflexivity of the Banach space $X$. Here, is a relevant example.

Example 4.7. Let $S$ be the unilateral backward shift on $c_{0}$. That is,

$$
S: c_{0} \longrightarrow c_{0},\left(\xi_{0}, \xi_{1}, \xi_{2}, \xi_{3}, \ldots\right) \mapsto\left(\xi_{1}, \xi_{2}, \xi_{3}, \ldots\right), \quad \text { where } x=\left(\xi_{0}, \xi_{1}, \xi_{2}, \xi_{3}, \ldots\right) \in c_{0}
$$

It is obvious that

$$
\begin{aligned}
& S^{*}: l^{1} \longrightarrow l^{1},\left(\eta_{0}, \eta_{1}, \eta_{2}, \eta_{3}, \ldots\right) \mapsto\left(0, \eta_{0}, \eta_{1}, \eta_{2}, \ldots\right), \quad \text { where } f=\left(\eta_{0}, \eta_{1}, \eta_{2}, \eta_{3}, \ldots\right) \in l^{1}, \\
& S^{* *}: l^{\infty} \longrightarrow l^{\infty},\left(\zeta_{0}, \zeta_{1}, \zeta_{2}, \zeta_{3}, \ldots\right) \mapsto\left(\zeta_{1}, \zeta_{2}, \zeta_{3}, \ldots\right), \quad \text { where } z=\left(\zeta_{0}, \zeta_{1}, \zeta_{2}, \zeta_{3}, \ldots\right) \in l^{\infty} .
\end{aligned}
$$

Then, (1) Both $S$ and $S^{* *}$ are not strictly cyclic. (2) $S^{*}$ is strictly cyclic on $l^{1}$. (3) The weakly closed algebra $\mathcal{A}_{S^{*}}$ generated by $S^{*}$ is semisimple. Therefore, $\mathcal{A}_{S}$ and $\mathcal{A}_{S^{* *}}$ are also semisimple by Lemma 3.3. (4) $A_{S^{*}}$ is a reflexive algebra, and $\mathcal{A}_{S^{*}}$ is hereditarily reflexive. (5) $\mathcal{A}_{S}$ is a reflexive algebra. (6) $\mathcal{A}_{S}$ is not hereditarily reflexive. (7) $\mathcal{A}_{S^{* *}}$ is a reflexive algebra, but $\mathcal{A}_{S^{* *}}$ is not hereditarily reflexive.

Proof. (2) Set $\beta_{0}=1, \beta_{n}=\prod_{k=1}^{n} w_{k}=1$. For any positive integers $m, n$, we have that $\left|\beta_{n} / \beta_{m} \beta_{n-m}\right|=1$. It follows that $\sup _{n, m}\left|\beta_{n} / \beta_{m} \beta_{n-m}\right|<\infty$. Since $\left\{\left|w_{n}\right|\right\}$ is monotonically decreasing, $S^{*}$ is strictly cyclic on $l^{1}$ by $[19]$ and $f_{0}=(1,0,0,0, \ldots)$ is a strictly cyclic vector for $\mathcal{A}_{S^{*}}$.

(3) Using the proof similar to the proof of (Example 4.4(3)), we have that $\|S\|=\left\|S^{*}\right\|=$ $\left\|S^{* *}\right\|=1$ and $r(S)=r\left(S^{*}\right)=r\left(S^{* *}\right)=1$. Since $S^{*}$ is unilateral forward shift on $l^{1}$, the spectra $\sigma(S)\left(=\sigma\left(S^{*}\right)=\sigma\left(S^{* *}\right)\right)$ of $S$ contains infinite complex numbers. Then, we have that the Jacobson radical $\operatorname{rad}\left(\mathcal{A}_{S}\right)=\{0\}$. Therefore, $\mathcal{A}_{S}, \mathcal{A}_{S^{*}}$ and $\mathcal{A}_{S^{* *}}$ are semisimple.

(4) $\mathcal{A}_{S^{*}}$ is reflexive by Lemma 3.7. $\mathcal{A}_{S^{*}}$ is hereditarily reflexive by Lemma 3.11.

(5) Suppose that $T \in B\left(c_{0}\right)$ and $\operatorname{Lat}(S) \subseteq \operatorname{Lat}(T)$. Since $T^{*} \in B\left(c_{0}^{*}\right)$ and $S^{*}$ has a strictly cyclic vector $f_{0}$, then there exists $T_{0} \in \mathcal{A}_{S}$ such that $T^{*} f_{0}=T_{0}^{*} f_{0}$. For any fixed $A \in \mathcal{A}_{S}$, let $T^{*} A^{*} f_{0}=B^{*} f_{0}$.

For each $x \in c_{0}$, let $M_{x}=\overline{\operatorname{span}}\left\{x, S x, S^{2} x, \ldots\right\}$, where the bar denotes the norm closure. Then, $M_{x} \in \operatorname{Lat}(S)$ and $M_{x} \in \operatorname{Lat}(T)$. We have that $\operatorname{Lat}\left(S \mid M_{x}\right) \subseteq \operatorname{Lat}\left(T \mid M_{x}\right)$ for every $x \in c_{0}$. It is obvious that $S$ is not locally algebraic, and there is $x \in c_{0}$ such that $\left\{x, S x, S^{2} x, \ldots\right\}$ is linearly independent. Let $x_{0} \in c_{0}$ such that $\left\{x_{0}, S x_{0}, S^{2} x_{0}, \ldots\right\}$ is linearly independent. Then, the algebra $\mathscr{A}_{S} \mid M_{x_{0}}=\left\{A \mid M_{x_{0}}: A \in \mathcal{A}_{S}\right\}$ has a separating vector $x_{0}$.

Let $\varphi$ be an arbitrary homomorphism from $\mathcal{A}_{S}$ to the complex field $\mathbb{C}$ and let $\mathcal{K}_{\varphi}=$ $\left\{A \in \mathcal{A}_{S}: \varphi(A)=0\right\}$ be the corresponding maximal ideal of $\mathcal{A}_{S}$. Define $\varphi^{*}: \mathcal{A}_{S}^{*} \rightarrow \mathbb{C}$ by $\varphi^{*}\left(A^{*}\right)=\varphi(A)\left(A \in \mathcal{A}_{S}\right)$. Then, $\mathcal{K}_{\varphi}^{*}=\left\{A^{*} \in \mathcal{A}_{S}^{*}: \varphi(A)=0\right\}$ be the corresponding maximal ideal of $\mathscr{A}_{S}^{*}$. For any $x \in c_{0}$, let $N_{x}=\mathcal{K}_{\varphi} x=\left\{A(x): A \in \mathcal{K}_{\varphi}\right\}$. Then, $N_{x} \subseteq M_{x}$ and $N_{x} \in$ $\operatorname{Lat}(S) \subseteq \operatorname{Lat}(T)$. So $T\left(N_{x}\right) \subseteq N_{x}$.

It is obvious that $\varphi(A) I-A \in \mathcal{K}_{\varphi}$ and $\varphi(A) x-A x \in N_{x}$. It follows that $\varphi(A) T x-T A x \in$ $N_{x} \subseteq M_{x}$. In particular, $\varphi(A) T x_{0}-T A x_{0} \in M_{x_{0}}$. Since $x_{0}$ is a separating vector for $\mathcal{K}_{\varphi}$, we have that $\varphi(A) T-T A \in \mathcal{K}_{\varphi}$. Then, $\varphi^{*}\left(A^{*}\right) T^{*}-T^{*} A^{*} \in \mathcal{K}_{\varphi}^{*}$ and $\varphi^{*}\left(A^{*}\right) T^{*}\left(f_{0}\right)-T^{*} A^{*}\left(f_{0}\right) \in$ $\mathcal{K}_{\varphi}^{*}\left(f_{0}\right)$, that is, $\varphi^{*}\left(A^{*}\right) T_{0}^{*}\left(f_{0}\right)-B^{*}\left(f_{0}\right) \in \mathcal{K}_{\varphi}^{*}\left(f_{0}\right)$. So, $\varphi^{*}\left(A^{*}\right) T_{0}^{*}-B^{*} \in \mathcal{K}_{\varphi}^{*}$ and $\varphi(A) T_{0}-B \in \mathcal{K}_{\varphi}$. Thus, $\varphi\left(\varphi(A) T_{0}-B\right)=\varphi(A) \varphi\left(T_{0}\right)-\varphi(B)=\varphi\left(T_{0} A-B\right)=\varphi\left(A T_{0}-B\right)=0$. Since $f_{0}$ is a separating vector for $\mathcal{K}_{\varphi}^{*}, T_{0} A-B \in \mathcal{K}_{\varphi}$ and $A T_{0}-B \in \mathcal{K}_{\varphi}$. Since $\varphi$ is arbitrary and $\mathscr{A}_{S}$ is semisimple, 
it follows that $B=T_{0} A=A T_{0}$, that is, $T^{*} A^{*} f_{0}=\left(A T_{0}\right)^{*} f_{0}=T_{0}^{*} A^{*} f_{0}$ for any $A \in \mathcal{A}_{S}$. Since $\mathscr{A}_{S}^{*} f_{0}=X^{*}, T^{*}=T_{0}^{*}$, and $T=T_{0} \in \mathcal{A}_{S}$. It follows that $\mathscr{A}_{S}$ is a reflexive algebra.

(6) By Lemma 3.14, the dual space of $A_{S}$ consists entirely of the maps $A \mapsto F\left(A^{*} f_{0}\right)$ $\left(A \in \mathcal{A}_{S}\right)$, where $F \in l^{\infty}$.

Since $\mathcal{A}_{S^{*}}$ is strictly cyclic, $\mathscr{A}_{S^{*}}$ and $\mathscr{A}_{S}$ are norm-closed, then $\mathscr{A}_{S^{*}}$ and $l^{1}$ are isomorphic as Banach spaces. Thus, $\mathcal{A}_{S}$ and $l^{1}$ are isomorphic as Banach spaces. It follows that the set of all weakly continuous linear functionals on $A_{S}$ is the set of all norm-continuous linear functionals on $A_{S}$.

Similar to Proposition 3.13, $A_{S}$ is hereditarily reflexive if and only if it is reflexive and every weakly continuous functional on $\mathcal{A}_{S}$ is of the form $A \mapsto f(A(x))\left(A \in \mathcal{A}_{S}\right)$, where $x \in c_{0}$ and $f \in l^{1}$. Since the Banach space $l^{1}$ is not reflexive, there exists $F \in l^{\infty}$ such that $F \notin J\left(c_{0}\right)$. It follows that $A_{S}$ is not hereditarily reflexive by the assertion.

(7) Using the proof similar to the proof of (5), we can prove that $\mathcal{A}_{S^{* *}}$ is a reflexive algebra. Using the proof similar to the proof of (6), we can prove that $\mathcal{A}_{S^{* *}}$ is not hereditarily reflexive.

\section{Acknowledgments}

The authors thank deeply the reviewers that led us to the articles [6, 7]. The authors would also like to express their hearty thanks to the referees for their very helpful comments and suggestions which improved the article greatly. $Q$. Chen would like to express deep gratitude to Professor Don Hadwin and Changli Tao for their hearty help during the work. This paper was supported by the National Nature Science Foundation of China (Grant 11071188).

\section{References}

[1] A. N. Loginov and V. S. Sulman, "The hereditary and intermediate reflexivity of $W^{*}$-algebras," Izvestiya Akademii Nauk SSSR, vol. 39, no. 6, pp. 1260-1273, 1975.

[2] A. N. Loginov and V. S. Sulman, "The hereditary and intermediate reflexivity of $W^{*}$-algebras," Mathematics of the USSR-Izvestiya, vol. 9, pp. 1189-1201, 1975.

[3] W. Arveson, "Operator algebras and invariant subspaces," Annals of Mathematics, vol. 100, pp. 433$532,1974$.

[4] E. A. Azoff, L. Ding, and W. R. Wogen, "Separating versus strictly separating vectors," Proceedings of the American Mathematical Society, vol. 124, no. 10, pp. 3135-3142, 1996.

[5] L. Ding, "Separating vectors and reflexivity," Linear Algebra and its Applications, vol. 174, pp. 37-52, 1992.

[6] D. W. Hadwin and E. A. Nordgren, "Subalgebras of reflexive algebras," Journal of Operator Theory, vol. 7, no. 1, pp. 3-23, 1982.

[7] D. W. Hadwin, "Algebraically reflexive linear transformations," Linear and Multilinear Algebra, vol. 14, no. 3, pp. 225-233, 1983.

[8] D. W. Hadwin and S.-C. Ong, "On algebraic and para-reflexivity," Journal of Operator Theory, vol. 17, no. 1, pp. 23-31, 1987.

[9] J. Kraus and D. R. Larson, "Some applications of a technique for constructing reflexive operator algebras," Journal of Operator Theory, vol. 13, no. 2, pp. 227-236, 1985.

[10] A. Lambert, "Strictly cyclic operator algebras," Pacific Journal of Mathematics, vol. 39, pp. 717-726, 1971.

[11] D. R. Larson, "Reflexivity, algebraic reflexivity and linear interpolation," American Journal of Mathematics, vol. 110, no. 2, pp. 283-299, 1988.

[12] A. I. Loginov and V. S. Sulman, "The hereditary and intermediate reflexivity of $W^{*}$-algebras," Doklady Akademii Nauk SSSR, vol. 212, pp. 810-812, 1973. 
[13] C. Peligrad, "Strictly cyclic operator algebras on Banach spaces," Integral Equations and Operator Theory, vol. 48, no. 4, pp. 557-560, 2004.

[14] C. L. Tao and S. J. Lu, "Separating vectors, separating functionals and reflexivity," Chinese Annals of Mathematics A, vol. 21, no. 4, pp. 513-516, 2000 (Chinese).

[15] D. A. Herrero and A. Lambert, "On strictly cyclic algebras, $D$-algebras and reflexive operators," Transactions of the American Mathematical Society, vol. 185, pp. 229-235, 1973.

[16] W. Rudin, Functional analysis, McGraw-Hill, New York, NY, USA, 1992.

[17] E. Kerlin and A. Lambert, "Strictly cyclic shifts on $l_{p}$," Acta Universitatis Szegediensis. Acta Scientiarum Mathematicarum, vol. 35, pp. 87-94, 1973.

[18] B. Barnes, "Operators with a strictly cyclic vector," Proceedings of the American Mathematical Society, vol. 41, pp. 480-486, 1973.

[19] M. R. Embry, "Strictly cyclic operator algebras on a Banach space," Pacific Journal of Mathematics, vol. 45 , pp. 443-452, 1973. 


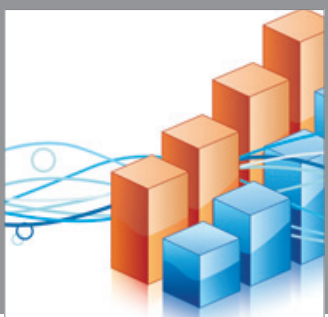

Advances in

Operations Research

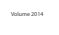

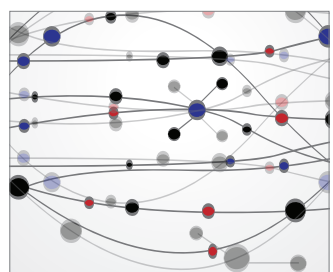

\section{The Scientific} World Journal
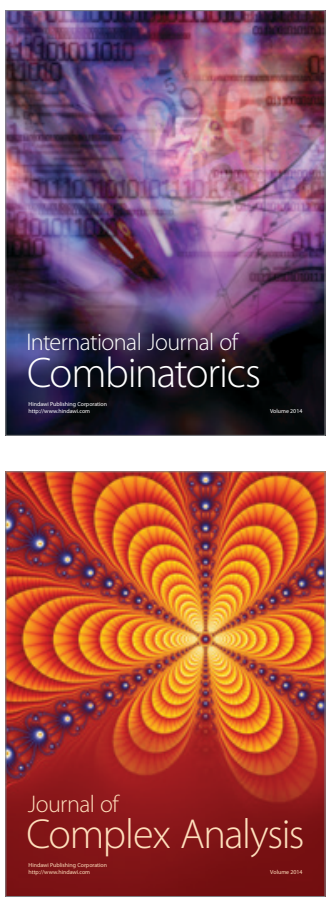

International Journal of

Mathematics and

Mathematical

Sciences
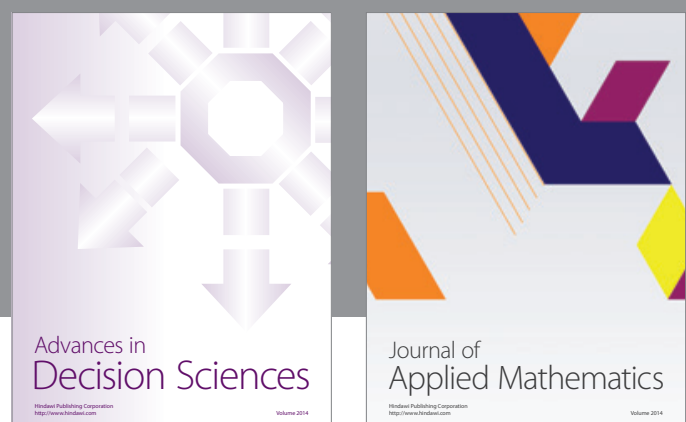

Journal of

Applied Mathematics
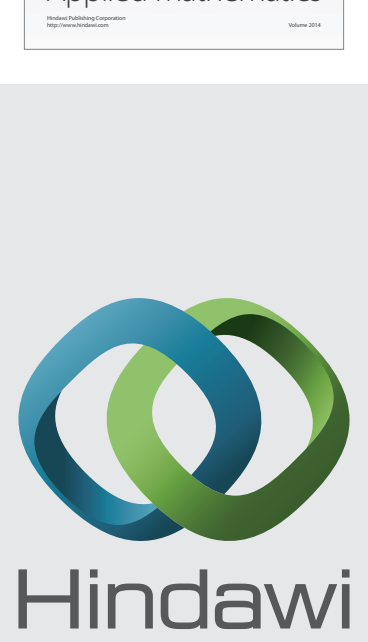

Submit your manuscripts at http://www.hindawi.com
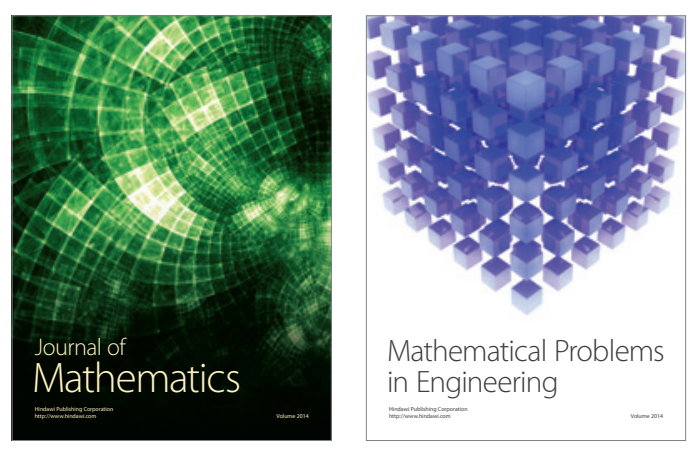

Mathematical Problems in Engineering
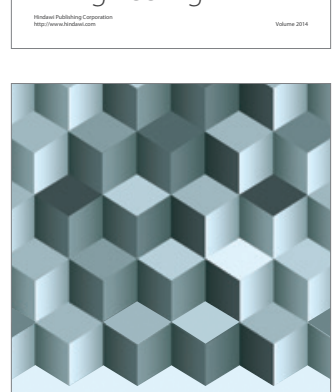

Journal of

Function Spaces
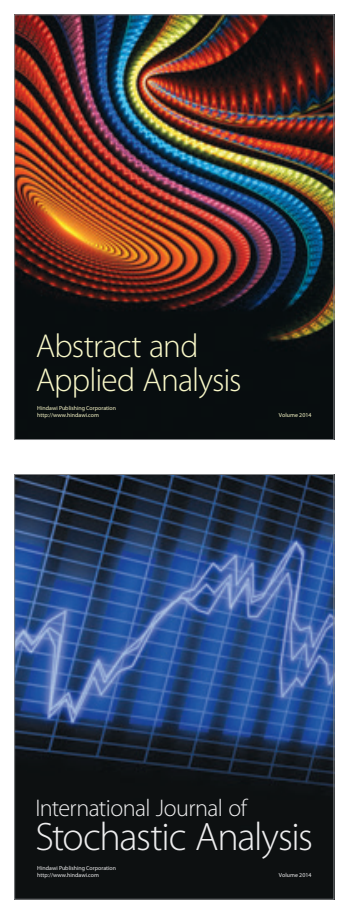

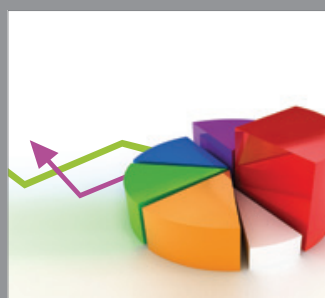

ournal of

Probability and Statistics

Promensencen
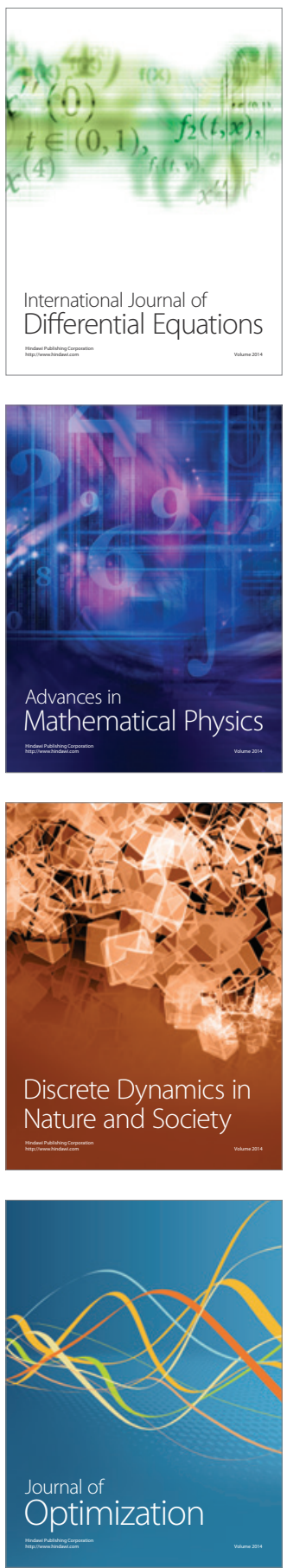